\title{
Stochastic modelling of particle aggregation
}

ARTICLE in INTERNATIONAL JOURNAL OF MULTIPHASE FLOW · DECEMBER 2015

Impact Factor: 2.06 · DOI: 10.1016/j.ijmultiphaseflow.2015.12.004

READS

15

3 AUTHORS, INCLUDING:

Salvador Navarro-Martinez

Imperial College London

48 PUBLICATIONS 533 CITATIONS

SEE PROFILE 


\section{Accepted Manuscript}

Stochastic modelling of particle aggregation

I. Pesmazoglou, A.M. Kempf, S. Navarro-Martinez

PII: S0301-9322(15)00274-8

DOI: 10.1016/j.ijmultiphaseflow.2015.12.004

Reference: $\quad$ IJMF 2316

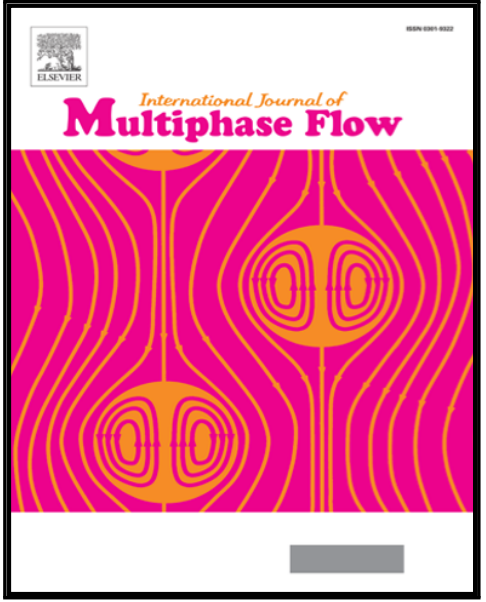

To appear in:

International Journal of Multiphase Flow

Received date:

11 June 2015

Revised date:

19 October 2015

Accepted date:

15 December 2015

Please cite this article as: I. Pesmazoglou, A.M. Kempf, S. Navarro-Martinez, Stochastic modelling of particle aggregation, International Journal of Multiphase Flow (2015), doi: 10.1016/j.ijmultiphaseflow.2015.12.004

This is a PDF file of an unedited manuscript that has been accepted for publication. As a service to our customers we are providing this early version of the manuscript. The manuscript will undergo copyediting, typesetting, and review of the resulting proof before it is published in its final form. Please note that during the production process errors may be discovered which could affect the content, and all legal disclaimers that apply to the journal pertain. 


\section{Highlights}

- The problems of simulating aggregation for large particle populations are highlighted.

- A stochastic multi-collision model to simulate aggregation is presented.

- The model presented allows for a direct coupling with an unsteady flow solution.

- The model is validated against analytic solutions for two aggregation kernels.

- The stochastic model is found accurate with respect to particle depletion and PSD evolution. 


\title{
Stochastic modelling of particle aggregation
}

\author{
I. Pesmazoglou
}

Department of Mechanical Engineering, Imperial College, London SW7 $2 A Z$

A. M. Kempf

Chair of fluid dynamics, Institute for Combustion and Gasdynamics, and CENIDE,

Center for Nano-Integration, Universität Duisburg-Essen, Duisburg, 47048

S. Navarro-Martinez*

Department of Mechanical Engineering, Imperial College, London SW7 $2 A Z$

\section{Abstract}

Aggregation is an inter-particle process which involves a multitude of different physicochemical mechanisms. In the present work, particles in the nano-scale are considered, with such concentration that renders their direct simulation as individual particles intractable. A stochastic aggregation model is presented for large particle populations in a Lagrangian framework. The model allows for simultaneous collisions between numerical parcels present in a certain volume of interaction (e.g. computational cell) and can be directly coupled to an unsteady numerical solver of a continuous flow. The model performance is evaluated against analytic solutions for a sum (Golovin) and constant aggregation kernel.

Keywords: Aggregation, Coagulation, Particles, Lagrangian, Aerosol

*Corresponding author:

Email address: s.navarro@imperial.ac.uk (S. Navarro-Martinez ) 


\section{Introduction}

The intersection of particle paths may lead to a collision event and an inelastic collision event to aggregation. A particle of volume $v$ (or mass $m$ ) is formed by the aggregation of two particles with volumes $v^{\prime}$ and $v-v$. However, its resulting volume is subject to the level of coalescence (also termed 'sintering' for solids), i.e. the extent to which the colliding-pair volumes merge. Depending on the phase of the particle (solid, liquid, or gaseous), the local thermodynamic conditions (e.g. temperature), and the collision process (angle of attack, relative velocity, etc.) the particle will attach to the 'receiver' particle differently, forming fractal aggregate structures (such as soot) or spherical aggregates. In the present study, particles in the nano-scale are considered and instantaneous coalescence of the colliding pair is assumed.

Binary droplet and particle collisions are an area of interest with applications ranging from meteorological to industrial processes. Meteorological phenomena such as cloud formation and raindrop formation drove research on experimental studies of aqueous coalescence in atmospheric air, see works by Adam and Lindblad (1968); Ashgriz and Poo (1990). Maximova and Dahl (2006) review the environmental implications of aggregation phenomena in the context of wastewater and gas emission treatment.

In the context of spray atomization, Gavaises et al. (1996) concluded that coagulation greatly influences the Particle Size Distribution (PSD) and consequently controls the particle relaxation times and velocities during injection.

Following the reports by Brauer et al. (2002); Finlayson-Pitts and Pitts (1997); Montes et al. (2004); Oberdorster (2001) on the effects of the size of 
aggregates on health, modelling of soot aggregates from combustion has also become an area of great importance, see Zucca et al. (2006).

Another example of industrial-scale nanoparticle aggregation is spray drying technologies (e.g. for the manufacturing of detergents and waterdissolvable powders), wherein aggregation governs the properties of the particles.

Aggregation can be both wanted and unwanted depending on the application. Aggregation is promoted in mining and spray drying applications to reduce wastage and make separation easier (e.g. enhance settling rates in gravity based separations). However, aggregation may reduce product quality by widening the PSD. In order to control the PSD, there has been an increasing effort to combine numerical modelling and experiments to investigate the aggregation process.

The objective of the present work is to develop a model for the aggregation process in the post-spray region using the spray probability density function (pdf) method of Willams (1958). The model focuses on applications particles with large number of particles, $>\mathcal{O}\left(10^{8}\right)$, such as nanoparticle formation, where one-to-one collision approaches are intractable and macroscopic statistical models are hence sought. The aim is to model macroscopic aggregation kernels in a Lagrangian framework in an implementation that represents the physics involved in the aggregation process, yet at the same time retains the advantages of the spray-pdf methodology. The proposed model can be directly coupled to an unsteady representation of the continuous flow field, such as Large Eddy Simulation, without any modification. In the present paper, the model is presented and validated against analytical 
solutions for different aggregation kernels.

\section{Modelling Aggregation}

\subsection{The aggregation kernel}

The aggregation process involves collisions of particles to form new ones with larger volumes. Consequently, both microscopic and phenomenological approaches can be sought.

In microscopic approaches each collision event is considered separately, such as the hard sphere collision model of Sundaram and Collins (1997); Yamamoto et al. (2001) and Yan et al. (2008). Microscopic models are used to understand the physics involved in the aggregation processes. Liao and Lucas (2010) categorise the numerous models for fluid particle coalescence processes and their relation to findings from binary (microscopic) collision experiments. However, in particle-laden flows in engineering applications, the number of particles, hereafter $N_{t o t}$, render microscopic approaches intractable.

Phenomenological approaches treat the collision process in a macroscopic framework. It is usually assumed that a collision event will result in the immediate coalescence of the two particles. Consider that $C_{i j}$ is the number of collisions per time and volume between two size classes $i$ and $j$ that form a new class $k$ with $v_{k}=v_{i}+v_{j}$. The joint probability of a collision to occur (and an aggregate to form) is quantified by the aggregation kernel (or frequency), $\beta_{i j}$, and the respective concentrations $N_{c, i}$ and $N_{c, j}$ such that:

$$
C_{i j}=\beta_{i j} N_{c, i} N_{c, j}
$$

The summation over all possible pairs $i, j$ leading to a size class $v_{k}$ gives rise to the Smoluchowski (1917) equation, also known as the Population Balance 
Equation (PBE). Considering only aggregation processes, the equation is:

$$
\frac{d N_{c, k}}{d t}=\frac{1}{2} \sum_{i+j=k} \beta_{i j} N_{c, i} N_{c, j}-N_{c, k} \sum_{i=1}^{\infty} \beta_{i k} N_{c, i} .
$$

Where, the kernel $\beta_{i j}$ incorporates the physico-chemical characteristics of the process. Macroscopic parameters of both the continuous and disperse phases may influence $\beta_{i j}$ depending on the physical mechanism that drives the aggregation process.

\subsubsection{Modelling the coupled kernel}

Solution methods for the flow-coupled aggrégation stem from those for the uncoupled kernel, namely: purely analytic, Method of Moments (MOM) and its variations (QMOM, HMOM and DQMOM), sectional methods, MonteCarlo (MC) methods, and combinations thereof. These can be solved either in an Eulerian or in a Lagrangian framework.

Purely analytic methods solve the aggregation integrals or the equivalent closed set of moment integrals. Such solutions are confined to a limited number of kernels (e.g. $\beta_{i j}=$ constant, $\beta_{i j}=v_{i}+v_{j}$, or $\beta_{i j}=v_{i} \times v_{j}$ ) for which the simple integrals can be directly evaluated, see for example Scott (1968), or used to close the PBE for specific initial PSDs (e.g. log-normal or mono-disperse). However, if the particles have a wide range of initial sizes, the aggregation kernel cannot be considered size-independent and analytic methods become intractable.

Eulerian frameworks used for the population balance solve a set of ODEs that result from a reconstruction of the PBE using a Method of Moments, or a discrete number of size classes. The reader may refer to the review of 
Yu and Lin (2010) for an overview of moment methods to solve nanoparticleladen flows.

Other widely used Eulerian approaches for the solution of the flow coupled PBE are discretization methods such as Yeoha and Tub (2006) and Miller and Garrick (2004). The number of 'bins' used for the discretization must represent the PSD accurately and a large number of bins might be required in problems where the PSD covers a wide range or widens signifieantly due to the particle processes, see Rigopoulos (2010). Azizi and Taweel (2010) suggested to adjust the PSD if its range requires expansion or contraction.

Stochastic/Monte Carlo methods simulate the aggregation process within a probabilistic framework; typically a Markov Jump process whereby two clusters merge with a given rate $C_{i j}$, see work by Wagner (2003). Stochastic and deterministic solutions of the PBE can be combined, for example Zou et al. (2010) combined a QMOM with an MC method. Rosner et al. (2003) reviews mixed moment and $\mathrm{MC}$ methods.

Direct Simulation Monte Carlo (DSMC) methods involve the solution of the PBE using stochastic particles that represent a single physical particle Eibeck and Wagner (2003). In practical applications, the number of particles suspended in the flow-field exceeds the computational capacity for a DSMC. The MC methods therefore simulate the evolution of a subset of the real particles using stochastic parcels. These parcels may represent a set of moments of the PBE, a set of discrete classes of the PBE, or a number of real particles representing a sampled size from the particle population.

Monte Carlo methods in the context of particle aggregation can be broadly categorised into two types of approaches, namely: constant number and 
constant-volume methods.

In constant-number MC, the number of simulated parcels does not change even though the real particle population is reduced due to aggregation events. The incentive is to reduce the statistical noise that would occur as a result of a decrease in simulation parcels after aggregation. The vacant spot of the particle array is refilled with the properties of a randomly selected particle that has survived. Therefore, the average mass of the simulated particles is also a random variable which depends on the mass of the (fictitious) new particle, which is randomly selected to fill the vacant position of the depleted particle; see work by Liffman (1992); Smith and Matsoukas (1998) and Lin et al. (2002).

Constant-number MC simulations use the same number of real particles per parcel and hence the tails of the PSD may not be sufficiently represented. Patterson et al. (2011) suggested that parcels must be distributed homogeneously over the PSD, i.e. even though regions might have low populations they must be represented by a sufficient number of parcels to statistically capture the poly-dispersity. Differential weighting of parcels may therefore mitigate errors that arise from a low resolution of the tails of the PSD.

\subsubsection{The Interaction Volume and Aggregation Time-step.}

In many practical applications, particles are in the nano-scale and their Brownian motion is the leading aggregation mechanism. The equivalent Brownian aggregation kernels are Eulerian in nature because the kernels are formulated in terms of particle concentration (i.e. number of particles per unit volume).

It is therefore important to choose an interaction volume $V_{I}$ that is phe- 
nomenologically accurate to estimate the particle concentration per unit volume. The volume of interaction defines the spatial region where the contained particles are allowed to interact, i.e. aggregate. Particles outside this spatial interaction volume cannot interact with those within $V_{I}$.

For example, Nijdam et al. (2004) use the model of Ruger et al. (2000) to calculate the collision frequency between two parcels with a given relative velocity $v_{r e l}$ nd relative volume of interaction $V_{I}=b_{1} x_{r e l}^{3}$, where $x_{r e l}$ is the relative distance between the parcels. The parameter $b_{1}$ is arbitrarily chosen, assuming a relation between the interacting volume and that of a sphere with a diameter equal to the inter-parcel distance. Guo et al. (2003) use $b_{1}=5$ to perform a base calculation and alter the value in a trial and error process. The two parcels merge if the calculated collision probability is greater than a critical value.

Zhao and Zheng (2013) couple the differentially weighted MC method of Zhao et al. (2009) and Zheng (2011) with a turbulent flow field. They assume that $V_{I}=V_{\text {cell }}$ and define a 'full coagulation rule' whereby all the real particles of a parcel $i$ collide instantaneously with the most probable partner $j$ given that $i$ contains less particles than $j$. The aggregation event is modelled using an accept/reject probabilistic coagulation rule in which a random number is generated and compared to a probability $\mathcal{P}_{\text {aggr }, i}$. The probability $/ \mathcal{P}_{\text {aggr }, i}$ is modelled as a Poisson distribution with a mean rate equivalent to the sum of all collision rates within $V_{\text {cell }}$, namely $C_{i}^{\prime}=\sum_{j} C_{i j}^{\prime}$. The prime indicates that the rate is modified according to the full coagulation rule of Zhao et al. (2009). All of the collisions are included in a single aggregation event with the most probable partner $j$ of parcel $i$. The partner 
is selected either using a cumulative probabilities method Liffman (1992) or with an acceptance-rejection method Lin et al. (2002).

A particle cloud (parcel), where an ensemble of real particles is represented by a single mean position, has the intrinsic disadvantage that the volume of the parcel depends on the internal spatial dispersion of the real particles. The level of internal dispersion may increase due to turbulence or decrease due to aggregation events. Litchford and Jeng (1991) suggest that each parcel position is represented by a normal distribution with a mean $\mu$ and standard deviation $\sigma$, which is representative of the parcel radius and is expressed in terms of a two-particle velocity correlation function. Alternatively, Johannessen et al. (2001), modelled the effeets of the flow field on the parcel by a 'dilution' factor.

The selection of the time-step $\Delta t$ for the integration of Eq. (2) should not influence the aggregation process. To de-couple the aggregation process from the dispersion process, the $\Delta t$ should be small enough that the relative distance between parcels does not change significantly within the time step (this can be achieved by applying a CFL-type condition when $V_{I}=V_{c e l}$ ). To accurately capture aggregation, the collision events should be in theory treated sequentially, since the kernels $\beta_{i j}=\beta_{i j}\left(v_{i}, v_{j}\right)$ change after every event In practice, a residence time $\tau_{\text {aggr }}$ is defined, see work by Zhao and Zheng (2013), which corresponds to the maximum coagulation rate of a parcel in a cell, $\tau_{\text {aggr }}=1 / \max _{i}\left(V_{\text {cell }} C_{i}^{\prime}\right)$. If $\Delta t>\tau_{\text {aggr }}$ then the aggregation is diyided into sub-steps $\Delta t_{\text {aggr }}<\tau_{\text {aggr }}$, where the rates are recalculated and aggregation events applied. 


\section{Numerical Modelling}

\subsection{The Spray-PDF Equation}

In the context of inertial particle dispersion, Williams (1958) introduced a conservation equation for the particle distribution function. The particle distribution function consists of the joint probability of the realization of $M$ variables that represent the state of the particulate phase: e.g. position, velocity, temperature, size, etc. For a set of $M$ independent variables the state vector of the $j^{\text {th }}$ particle for a given location in space $\mathbf{x}$ and time $t$ is $\boldsymbol{\Phi}^{j}=\left\{\phi_{1}^{j}(\mathbf{x}, t), \ldots, \phi_{M}^{j}(\mathbf{x}, t)\right\}$. The spray-pdf describes the evolution of the the phase space $\boldsymbol{\Psi}$ of the random variables $\psi_{i}$ (with realizations $\boldsymbol{\Phi}^{j}$ for every $j$-indexed particle) of an ensemble of $N$ particles. The mathematical formulation of the spray-pdf equation lies outside the scope of this work; the interested reader may refer to the original work by Klimontovich (1969) and Bini (2006).

By equivalence, the spray-pdf can be reformulated as a trajectory problem to describe the evolution of the random variables $\psi_{i=1 \ldots M}$, for a set of $j$ indexed stochastic parcels (samples), with a set of Stochastic Differential Equations, for a complete description see Pesmazoglou et al. $(2013,2014)$ :

$$
d \psi_{i}^{j}=A_{i}(\boldsymbol{\Psi}) d t+B_{i k}(\mathbf{\Psi}) d W_{t, k}+J_{i}(\mathbf{\Psi}) \delta N_{d t}^{j, \lambda}
$$

where $A_{i}$ is the drift term, $J_{i}$ the jump term, and $\delta N_{d t}^{j, \lambda}$ is the $j^{\text {th }}$ increment of a stochastic counting process of rate $\lambda$. Any continuous random forcing can be added both to the spray-pdf and the trajectory problem with a Brownian diffusion term quantified by an equivalent diffusion tensor $B_{i k}$. 
In the present work, aggregation is investigated in a Lagrangian framework for particle populations that render the numerical representation of all particles on a one-to-one (particle to parcel) basis intractable. Each numerical parcel represents a set of real particles with the same average trajectories.

\subsection{Aggregation Model}

Aggregation is incorporated into the spray-pdf by the addition of particle number concentration and volume (or diameter assuming constant particle density) in the set of Lagrangian equations described by Eq. (3). In aggregation processes, the number of real particles and the average size represented by a parcel do not remain constant; and both change due to inter-particle interactions. Therefore a Lagrangian trajectory for each property has to be formulated and solved.

The analysis that follows focuses on particles in the nano-scale range, for which the Brownian aggregation mechanisms dominate, and the respective aggregation kernels depend on particle concentration (which is of Eulerian nature). The particle concentration can be obtained by the approach of Johannessen et al. (2001), where the trajectory directly represents the particle concentration of a stochastic parcel. Alternatively, the real particle number of a parcel can be traced and the equivalent concentrations for the evaluation of the Brownian kernels can be retrieved by defining a volume of interaction $V_{I}$ (see Section 2.1.1).

In the Lagrangian context of this work, the real particle population per parcel is tracked in order to maintain a more intuitive representation of the particulate phase and to allow for a direct extension of the spray-pdf. The 
following stochastic trajectory is therefore formulated:

$$
d n_{\text {int }}=-\dot{n}_{\text {coll }} d t
$$

where $n_{\text {int }}$ is the number of real particles in a given parcel, $\dot{n}_{\text {coll }}$ is the rate of collisions of real particles of the parcel. The number of collisions $n_{\text {coll }}$ that cause a change $d n_{\text {int }}$ in a given time $d t$ is modelled along the lines of a jump process with time increments $d t$ and a mean rate $\tau_{\text {aggr }}$.

The parcel concentration is defined as:

$$
n_{p}(t)=\frac{n_{\text {int }}(t)}{V_{I}}
$$

and the Eulerian particle number concentration for a certain sample volume $V_{\text {samp }}$ centered at a position $\mathbf{x}$ is:

$$
N_{c}(\mathbf{x}, t)=\sum_{i=1}^{\forall i \in V_{\text {samp }}} \frac{n_{\text {int }, i}}{V_{\text {samp }}},
$$

For simplicity the sampling volume is chosen to be that of a cell, i.e. $V_{\text {samp }}=$ $V_{\text {cell }}$.

The volume of interaction $V_{I}$ for the aggregation process is modelled by a cloud-cuboid (see two-dimensional sketch in Figure 1). The interaction volume between two parcels $i, j$ is equal to the length of the two aligned parcels multiplied by a height and width equal to that of the largest parcel, viz. :

$$
V_{I, i j}=\frac{1}{2}\left(r_{c, i}+2 x_{r e l}+r_{c, j}\right) \max \left(r_{c, i}, r_{c, j}\right)^{2} .
$$

Note that conceptually the interaction volume defines a certain spatial region wherein real particles aggregate. In essence, the number of particles 


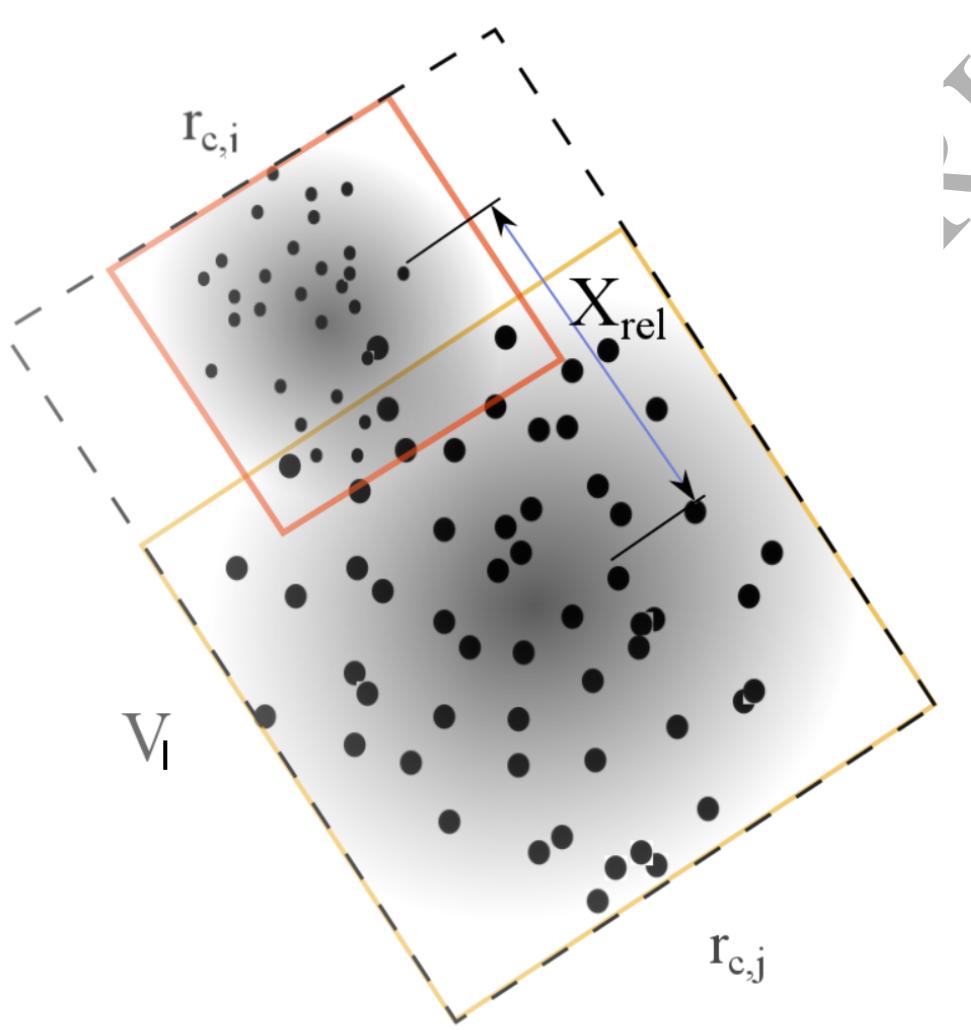

Figure 1: Interaction volume of two interacting parcels $i, j$. The interaction volume is indicated by the dashed line. 
divided by the equivalent interaction volume define the local concentration of particles $\left(\# / m^{3}\right)$. Particles that lie outside this volume are considered passive (physically too far to collide within a time-step).

To estimate the side of each parcel cube, $r_{c}$, particle dispersion inside the parcel is assumed to behave similarly to molecular diffusion in the carrier phase. The cube side is then

$$
r_{c}=n_{i n t}^{\frac{1}{3}} \mathrm{Kn}_{f} d_{p}
$$

where $\mathrm{Kn}_{f}$ is the Knudsen number of the carrier-fluid phase (based on mean free path, $\ell$, and molecule size) and $d_{p}$ is the mean particle diameter. The mean distance between each particle is clearly $\mathrm{Kn}_{f} d_{p}$. If the particle dispersion within a parcel is primarily due to Brownian diffusion, the equivalent parcel size must be smaller than the smallest fluid carrier mixing-scale $r_{c}<l_{B}$, where $l_{B}=\eta_{k} / \mathrm{Sc}^{1 / 2}$ is the Batchelor scale; with $\eta_{k}$ the Kolmogorov scale and Sc the Schmidt number of the carrier phase.

The aforementioned assumption sets a maximum limit to the number of internal particles in the parcel, namely:

$$
n_{\text {int,max }} \leq \max \left[\left(\frac{l_{B}}{\mathrm{Kn}_{f} d_{p}}\right)^{3}, 1\right]
$$

Figure 2 displays the maximum number of particles per parcel versus diameter, for nanoparticle dispersion in ambient air. This limit of course, has to be offset by the computational demands of the simulation and upper/lower limits can be set.

The diffusion mechanism of nano-particles in fluids is different from both the Brownian motion and the molecular mechanisms and strongly depends 


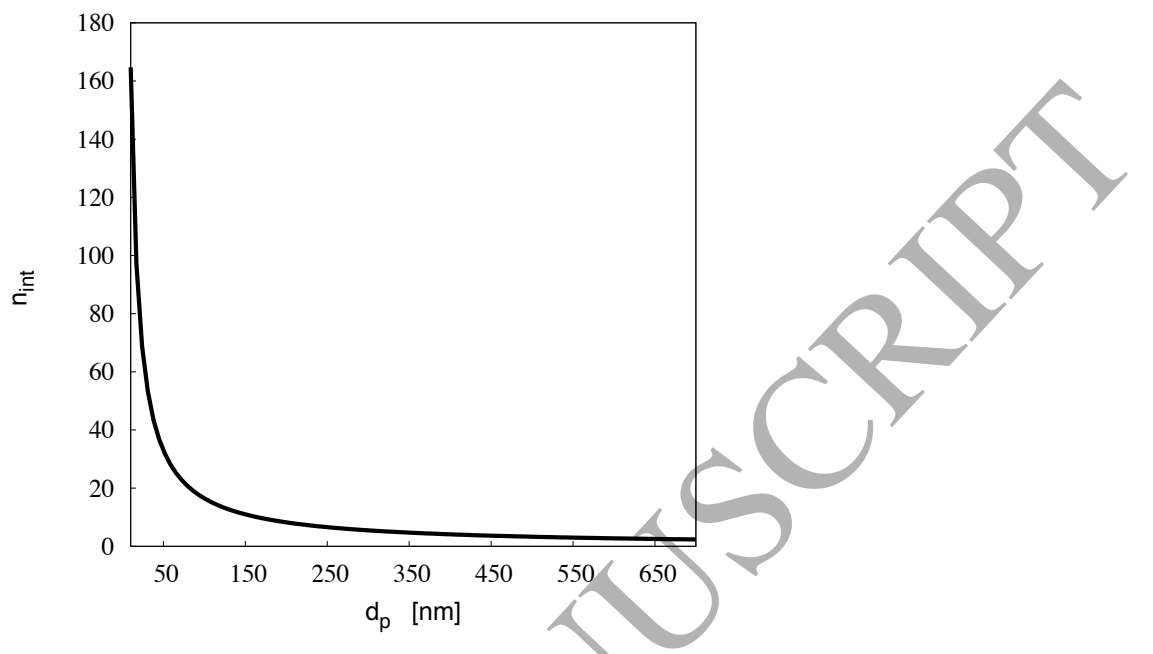

Figure 2: Maximum number of real particles represented by one parcel as a function of particle diameter. With $\eta_{k}=0.28 \mathrm{~mm}, \mathrm{Sc}=1$ and $\mathrm{Kn}_{f=}=170$

on the particle diameter. For small nano-particles, $d_{p} \sim \ell$, the particles can be considered in the free-molecular regime and their diffusion dynamics approximated by the Chapman-Enskog theory, see Li and Wang (2003). For larger particles, the diffusion coefficient can be determined by the EinstenStokes relation, that predict values as low as $10^{-9} \mathrm{~m}^{2} / \mathrm{s}$. For such small diffusivity coefficients, Sc $>>1$ and Eq. (9) predicts $n_{\text {int }, \max }=1$.

The $n_{\text {int,max }}$ limit offers a number of advantages from a phenomenological perspective:

- Particles inside the parcel are not influenced by vortical motions and their concentration can be considered analogous to that of molecules in the gas phase (gravitational effects can be considered negligible).

- The relative internal parcel dispersion will not increase due to fluid 
structures and the particles will be transported as an ensemble.

- Nanoparticles PSD usually have log-normal distributions wherein large diameters have low populations, this is reflected in the limit (9)

In the present multi-collision model, the particles of a parcel may collide with a number of different parcels. If every collision event created an additional parcel, then the number of parcels in the simulation would increase to an intractable number. Therefore, it is assumed that the aggregated particles from a colliding parcel-pair are added to one of the two colliding parcels. The parcel that has the larger mean particle diameter accommodates the aggregated particles and is termed 'receiver' whereas the other parcel is termed 'donor'. To simplify the multi-collision process the further assumption is made:

- More than one donor particle may collide with a single particle of the receiver parcel but not vice-versa.

This assumption suggests that if a large population of small particles (represented by a donor parcel $i$ ) interacts with a few large particles (receiver $j$ ), the large particles may experience more than one collision event; the number of collisions $n_{\text {coll }, i j}$ may be greater than $n_{i n t, j}$. However, the total number of collision events is limited to the internal population of the donor parcel, i.e. for the same notation of $i, j$ parcels:

$$
\sum_{j}^{\forall j \in V_{\text {cell }}} n_{\text {coll }, i j}=\min \left(\sum_{j}^{\forall j \in V_{\text {cell }}} n_{\text {coll }, i j}, n_{\text {int }, i}\right) .
$$

The suggested approach prevents collisions from being double counted since although $\beta_{i j}=\beta_{j i}$, the number of collisions $n_{\text {coll }, i j} \neq n_{\text {coll }, j i}$. The compu- 
tational advantage of this method is that a second loop to find the most probable partner is not required; and array operations can be employed by formulating the tensor $n_{\text {coll }, i j}$.

The novelty in the proposed model is that the particles are allowed to collide with any set of particles (including particles within the same parcel) within $V_{\text {cell }}$ instead of colliding with a single most probable partner. The size of the newly-formed aggregates is described by the ensemble average size (average over total internal particles) of the receiver parcel and if insufficient events are simulated, a poly-disperse PSD may be smoothened. The condition of Eq. (9) partly mitigates such errors as parcels with large-size particles have lower $n_{\text {int }}$.

The collision tensor $n_{\text {coll }, i j}$ gives the number of collision events that occur between parcel $i$ and parcel $j$. A probabilistic approach is used for the simulation of such events, whereby the probability of $n_{\text {coll }, i j}$ collisions to take place between two parcels $r$ and $j$ within a volume $V_{I}$ and in a time interval $t$ is given by:

$$
\mathcal{P}_{a g g r}\left(n_{c o l l, i j}, t\right)=\frac{1}{n_{\text {coll }, i j}} \frac{t}{\tau_{\text {aggr }}},
$$

where $\tau_{\text {aggr }}$ is the aggregation residence time:

$$
\tau_{\text {aggr }}^{-1}=\frac{\beta_{i j} n_{i n t, i} n_{i n t, j}}{V_{I}} .
$$

A linear increase of probability with $t$ is assumed and the measure $\mathcal{P}_{\text {aggr }}\left(n_{\text {coll }, i j}, t\right)$ is the CDF representing the probability of $n_{c o l l, i j}$ events to occur at any time in the range $[0: t]$. The division by $n_{\text {coll }, i j}$ normalizes the probability to a maximum of $\mathcal{P}_{\text {aggr }}\left(n_{\text {coll }, i j}, t\right)=1$ for any number of collision events. Note that $V_{I}$ may be equal to $V_{\text {cell }}$ for any $i, j$ pair, or evaluated separately for each $i, j$ pair using Eq. (7). 
By sampling a random number, $R n$, from an uniform distribution in the range $0 \rightarrow 1$, the equivalent $i j$ element of the collision tensor for a time-step $\Delta t$ is constructed as :

$$
n_{\text {coll }, i j}= \begin{cases}\text { floor }\left(\frac{\Delta t}{\tau_{\text {aggr }}}+R n\right), & \text { if } v_{i}<v_{j} \\ 0, & \text { otherwise }\end{cases}
$$

The function 'floor' produces the integer part of a real number. Equation (13) states that the total number of collisions in the direction $i \rightarrow j$ during an elapsed time $\Delta t$ for $v_{i}<v_{j}$ is equal to the integer (or definite) number of collisions plus the floor of the sum of the remáinder and $R n$. If for example $P_{\text {aggr }}(1, \Delta t)=\Delta t / \tau_{\text {aggr }}=0.8$ then there is an $80 \%$ probability that $R n \geq 0.2$ and therefore that a collision will take place. Note that $n_{\text {coll }, j i}=0$ if $v_{i}>v_{j}$. To optimize the number of operations required and also to include the cases where $v_{i}=v_{j}$ without double-counting, the collision array $n_{\text {coll }, i j}$ (13) for all pairs $i-j$ and internal collisions $i-i$ is constructed using a particle sorting algorithm per cell.

To ensure that the aggregation events are sufficiently resolved in time, the time-step limit of Zhao and Zheng (2013) is used:

$$
\Delta t_{\text {aggr }} \leq \min _{\forall i \in \text { Domain }}\left[\left(\sum_{j}^{\forall j \in V_{\text {cell }}} \frac{\beta_{i j} n_{\text {int }, i} n_{\text {int }, j}}{V_{I, i j}}\right)^{-1}\right] .
$$

This criterion implies that the time-step is limited by the maximum total aggregation rate of all parcels in the domain.

Figure 3 summarizes the collision events graphically. Some of the smallsize particles of the donor parcel (blue in the online version) collide with, and therefore are added to, the total mass of the receiver/donor parcel (grey in the 


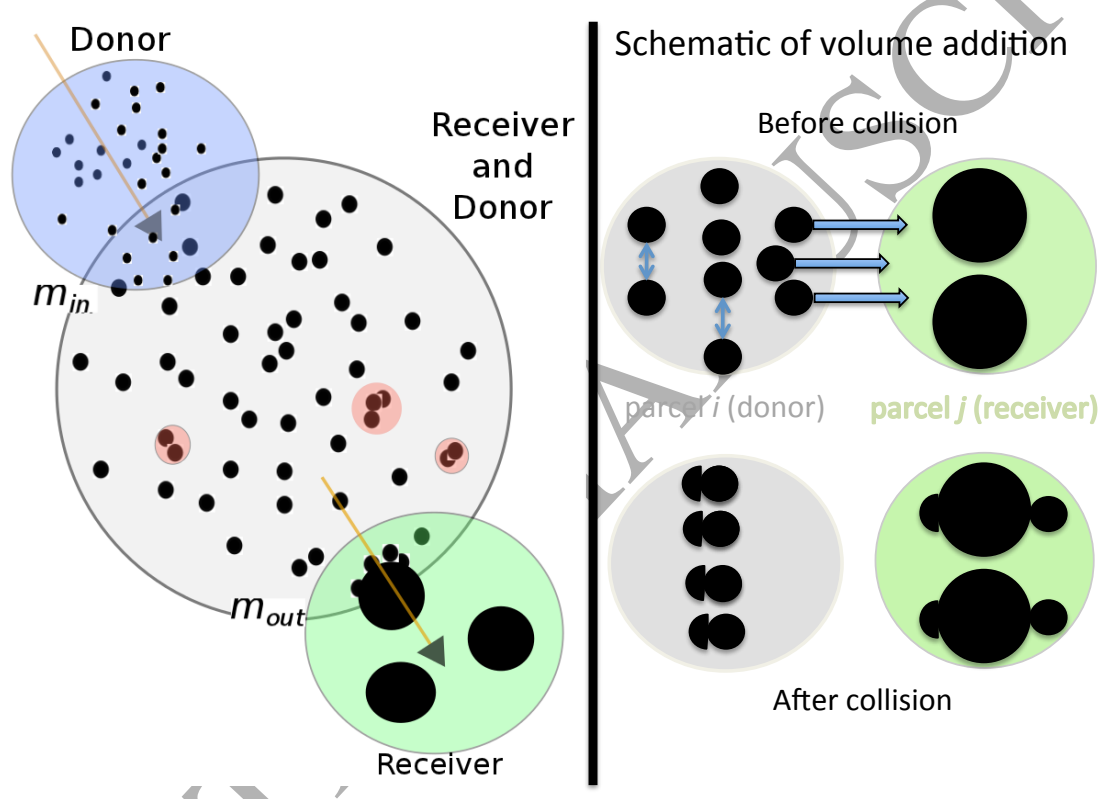

Figure 3: The mass exchange and collision events assuming that donor parcels always contain smaller particles than receiver parcels.

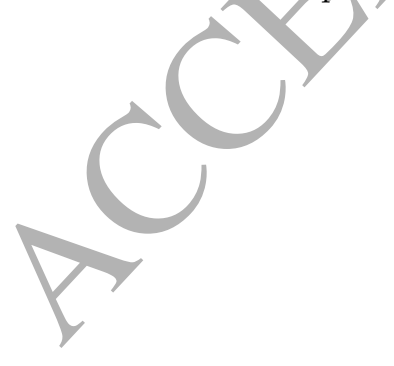


online version) The internal number of particles of the donor/receiver parcel remains unchanged and therefore their average mass increases. However, the size of the particles of the donor parcel remains the same, since the outward mass-flux is balanced by a decrease in the internal number of particles. The parcel undergoes a number of internal collisions, which do not change its total mass, but since the internal number of particles drops, the average mass per particle increases, and so does their average size (assuming constant density). The collisions with the larger particles of the receiver parcel (green in the online version of Fig. 3) cause a net outward flux of mass from the donor/receiver parcel towards the receiver parcel. Although the total mass of the receiver/donorparcel drops, the average mass of the internal particles is unchanged as the population of the parcel also decreases. Finally, the receiver parcel will increase its total and average mass as $n_{\text {int }}$ is constant.

For every parcel $i$ the effect of the collision events are incorporated to the equivalent trajectories of each partner $j$. Note that a constant particle density $\rho_{p}$ is assumed for all the following calculations.

The change in particle mean volume of a parcel $j$ is updated using a weighted sum of the colliding particle volumes (see schematic in Fig. 3). It is therefore assumed that each particle of the receiver parcel will increase in average size according to:

$$
\left[d v_{j}\right]_{a g g r, i j}=\frac{n_{\text {coll }, i j}}{n_{\text {int }, j}} v_{i}
$$

Therefore the equivalent trajectory for the average parcel volume of the receiver $j$ due to collisions with all donor parcels $i$ is:

$$
d v_{j}=\sum_{\forall i \in V_{\text {cell }}}\left[d v_{j}\right]_{a g g r, i j}
$$


Finally, having updated the aforementioned properties, the new internal particle number of each parcel is updated according to:

$$
d n_{\text {int }, i}=-\sum_{\forall j \in V_{\text {cell }}} n_{\text {coll }, i j} .
$$

Figure 4 is a flow-chart which briefly outlines the aforementioned methodology. Assuming that within the CFD time-step the particles do not change cells, the aggregation process can be readily re-iterated for a shorter timestep following the steps outlined previously. A separate sensitivity test for the aggregation time-step was carried out in stationary (no flow) conditions. In its generality, the outlined multi-collisional approach for stochastic parcels can be used with different aggregation mechanisms and kernel modifications to include a fractal shape.

\section{Results}

For the preliminary validation and development of the aggregation model outlined in section 3.2, the analytic solutions of the PBE for two aggregation kernels are used. The aim is to investigate the performance of the multicollision model with respect to particle concentration depletion, particle total mass conservation, and the PSD evolution. In addition, the influence on the aforementioned statistics of the aggregation time-step $\Delta t_{\text {aggr }}$ and internal particles per parcel $n_{\text {int }}$ selected is investigated. The particle depletion process is investigated using a constant aggregation kernel $\beta_{i j}=\beta_{c}$ and the PSD evolution using both a constant and a sum kernel $\beta_{i j}=\beta_{c}\left(v_{i}+v_{j}\right)$, also termed the Golovin (1963) kernel. The simulation time varied from a few CPU minutes to $40 \mathrm{CPU}$ hours in the cases with the smallest number of real particle per parcel (termed 'finest' cases). 


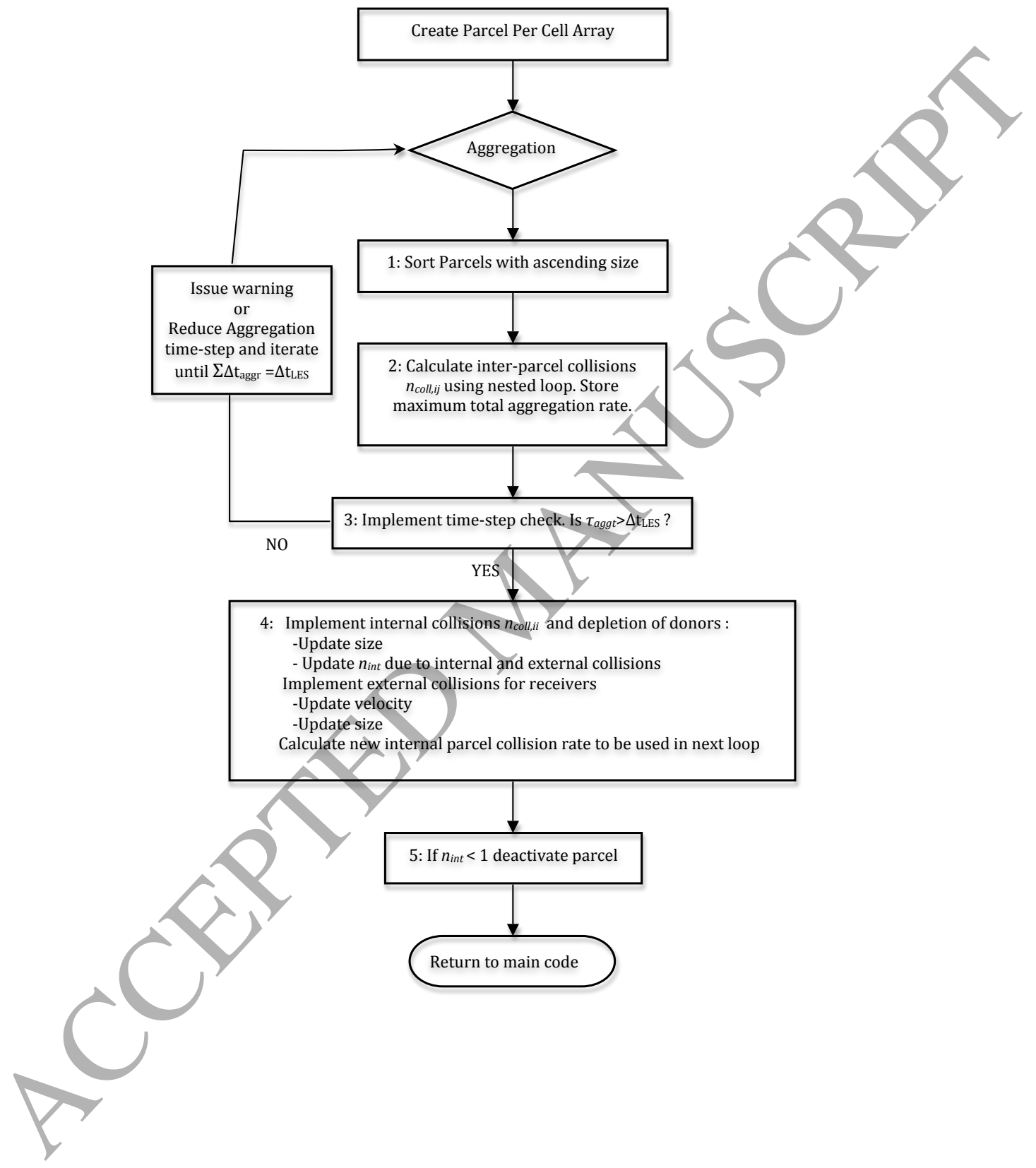

Figure 4: Flow chart of multi-collision aggregation model. 


\subsection{Particle depletion due to aggregation}

For the uncoupled PBE, with a constant aggregation kernel, an equation for the total particle concentration $N_{c}$ can be retrieved by summing over all the classes of Eq. (2), by:

$$
\begin{aligned}
\frac{d N_{c}}{d t} & =\sum_{k=1}^{\infty} \frac{1}{2} \sum_{i+j=k} \beta_{c} N_{c, i} N_{c, j}-\sum_{k=1}^{\infty} N_{c, k} \sum_{i=1}^{\infty} \beta_{c} N_{c, i} \\
& =\frac{\beta_{c}}{2} \sum_{k=1}^{\infty} \sum_{i+j=k} N_{c, i} N_{c, j}-\beta_{c} N_{c}^{2} \\
& =-\frac{\beta_{c}}{2} N_{c}^{2} .
\end{aligned}
$$

Integration of the above expression yields:

$$
N_{c}(t)=\frac{N_{0}}{1+t^{*}}
$$

Where $N_{0}$ is the initial particle concentration at $t=0$ and $t^{*}=t / \tau_{\text {aggr }}^{\prime}$ is a dimensionless time with $\tau_{\text {aggr }}^{\prime}=2 /\left(\beta_{c} N_{0}\right)$ (different from the aggregation residence time of Eq. (12). For the complete derivation, the reader can refer to Friedlander (2000).

Making use of Eq. (18), it is possible to investigate the proposed MC aggregation model with respect to total particle depletion and mass conservation. For eyery parameter investigated, a minimum of $s_{n, t o t} \geq 10$ simulations were carried out to investigate convergence and performance. The ensemble-averaged errors for a generic variable $\phi$ are defined as:

$$
\left\langle\left|\epsilon_{\phi}\right|\right\rangle_{s_{n}}=\frac{1}{s_{n, t o t}} \sum_{s_{n}=1}^{s_{n, t o t}} \frac{\phi^{\text {analytic }}-\phi^{M C, s_{n}}}{\phi^{\text {analytic }}}
$$

For simplicity, the dimensionless ratio of initial internal parcel population 


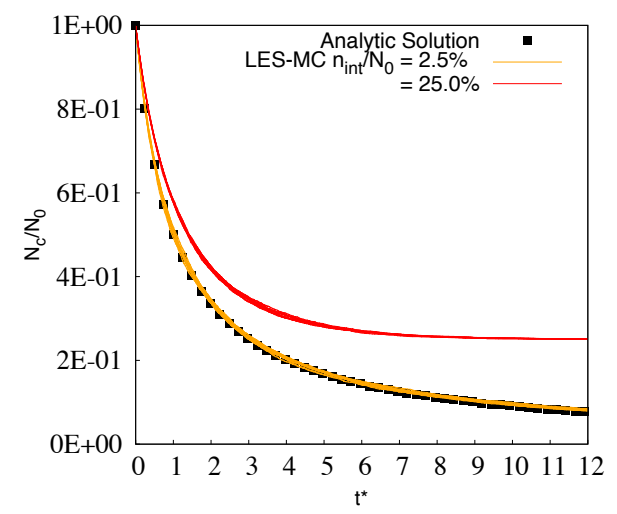

Figure 5: Total particle concentration versus dimensionless time for the same initial particle concentration using $n_{0}^{*}=2.5 \%$ and $25 \%$.

$n_{\text {int }}$ to initial particle population $N_{0} V_{\text {cell }}$ is defined as:

$$
n_{0}^{*}=\frac{n_{\text {int }} \mid t_{\bar{y}}}{N_{0} V_{\text {cell }}}
$$

Figure 5 compares the analytic solution of the particle depletion for two cases. In the first case the initial concentration $N_{0}$ is represented by parcels with an internal population equivalent to $n_{0}^{*}=2.5 \%$, whereas in the second case $n_{0}^{*}=25 \%$ (e.g. for a total of 1000 particles the first case has parcels with $n_{\text {int }}=25$ and the second has parcels with $n_{\text {int }}=250$ ). From Fig. 5 it is clear that neglecting internal collisions may lead to significant errors in the particle depletion statistics; $\left\langle\left|\epsilon_{N_{c}}\right|\right\rangle \sim 200 \%$ and $8 \%$ for the $n_{0}^{*}=25 \%$ and $2.5 \%$ cases respectively.

Figure 6 shows the variation of particle concentration including internal collisions for four different initial $n_{\text {int }}$, namely: $n_{0}^{*}=25 \%, 2.5 \%, 0.25 \%$ and $n_{\text {int }}=1$. Good agreement is found with the analytic solution for all four cases. Comparing Figs. 5 and 6, it becomes clear that internal collisions 

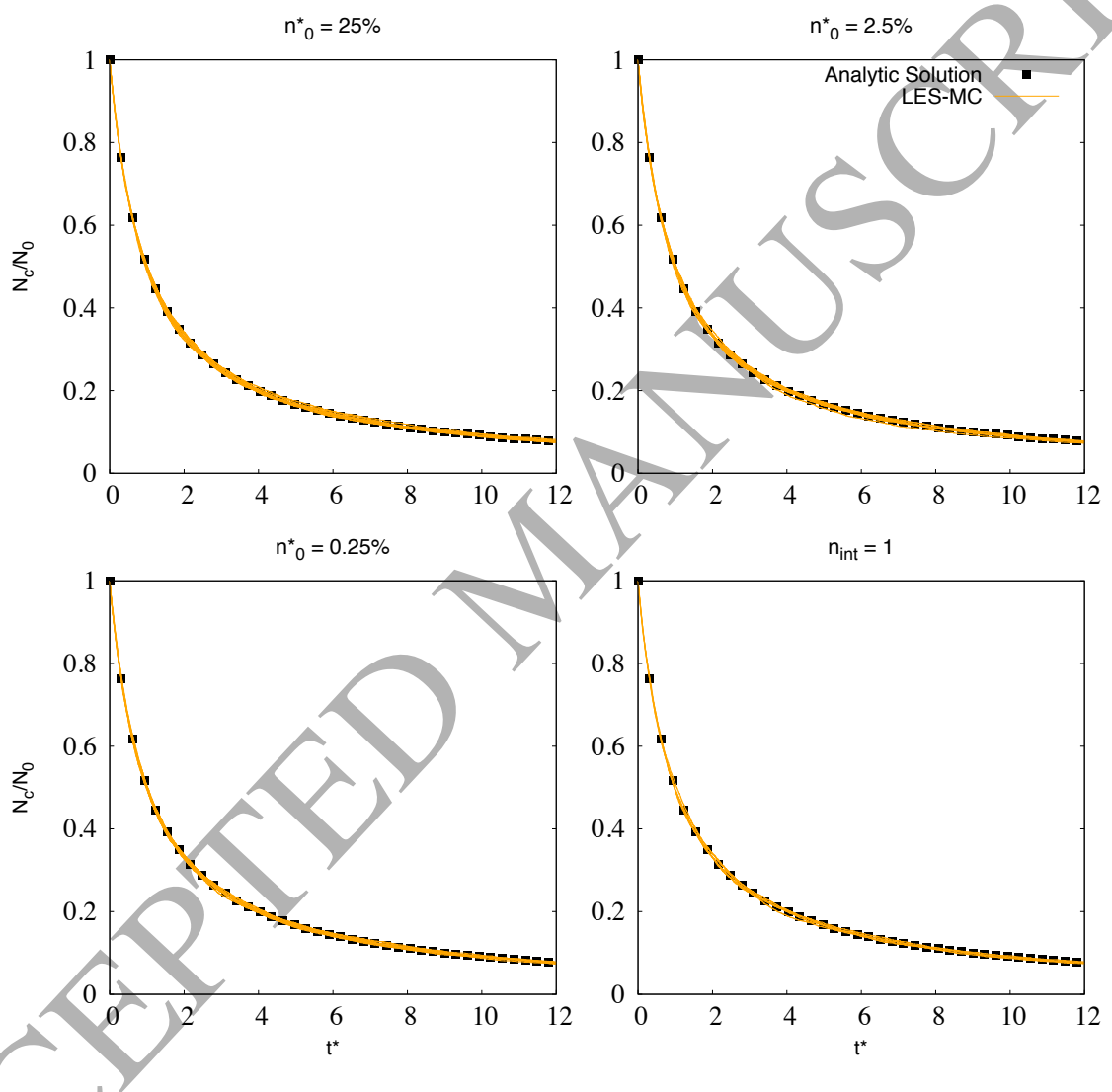

Figure 6: Total particle concentration versus dimensionless time for the same initial particle concentration using $n_{0}^{*}=25 \%, 2.5 \%, 0.25 \%$ and $n_{\text {int }}=1$. 


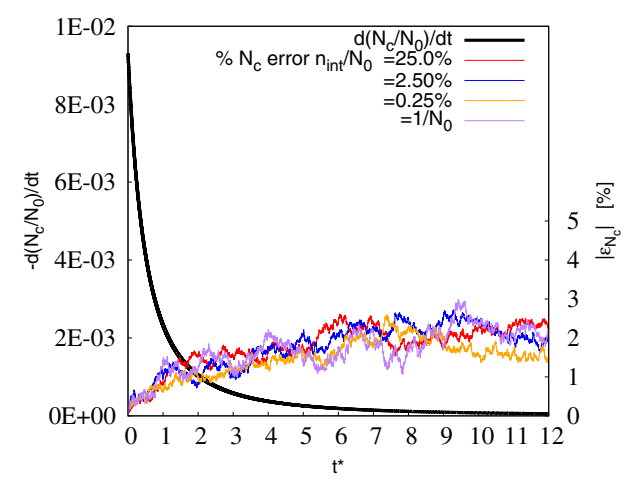

(a) Rate of particle depletion (left)

Concentration error (right).

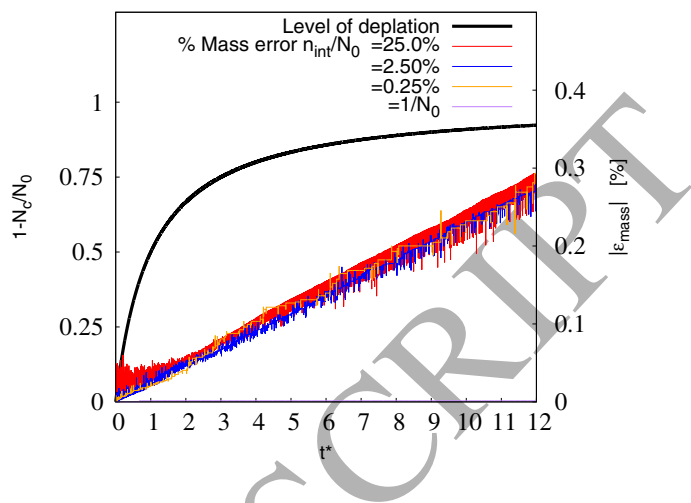

(b) Level of particle depletion (left)

Mass error (right).

Figure 7: Error levels for different pareel internal populations $n_{\text {int }}$.

need to be included, when parcels representing many physical particles are used, in order to accurately capture the depletion statistics. These results suggest that the initial parcel internal population does not affect the depletion characteristics when a constant aggregation kernel is used ${ }^{1}$.

Figures $7 \mathrm{a}$ and $7 \mathrm{~b}$ show the error, see Eq. (20), in total particle concentration and in total particle mass, respectively. Once the rate of depletion $d\left(N_{c} / N_{0}\right) / d t$ is very low, the steady-state concentration error $\left\langle\left|\epsilon_{N_{c}}\right|\right\rangle$ is $0-2 \%$ for all initial $n_{\text {int }}$ investigated. The mass error $\left\langle\left|\epsilon_{\text {mass }}\right|\right\rangle$ for all cases where $n_{\text {int }}>1$ exhibits a similar monotonic increase with time. However, the ab-

${ }^{1}$ Note that a constant kernel suggests that a single parcel could represent the total population of particles. Since at this point the PSD evolution is not considered, it is therefore expected that the mean volume and number concentration characteristics that a single parcel depicts are sufficient to represent the particle depletion process for a constant kernel. 
solute error does not exceed $0.3 \%$ and is negligible in systems with a low aggregation residence time. In the case where $n_{\text {int }}=1$, the error is virtually zero. The mass error probably stems from rounding-off errors that appear due to the weighted update of the parcel volumes; see Eq. (15). Roundoff errors accumulation also explains the observed monotonic increase of the mass error (see Fig. 7b)

In the simulations, the dimensionless time-step $\Delta t_{\text {aggr }}^{*}=\Delta t_{\text {aggr }} / \tau_{\text {aggr }}^{\prime}$ is of the order of $10^{-3}$. Figure 8 presents the variation of particle concentration with $\Delta t_{\text {aggr }}^{*}$ in the range $10^{-1}-10^{-5}$. The largest discrepancies between the analytic and $\mathrm{MC}$ concentrations are for the coarsest temporal discretization. For $\Delta t_{\text {aggr }}^{*} \leq 10^{-2}$, the particle concentration evolving in time is accurately represented by the model. The following empirical stability condition can be then used to de-couple the aggregation process:

$$
\Delta t<\alpha_{a g g r} \tau_{a g g r}^{\prime}
$$

where $\alpha_{\text {aggr }} \sim \mathcal{O}\left(10^{-2}\right)$

Figure 9a shows the error in total particle concentration for different aggregation time-steps. The concentration error seems to be fairly unaffected by the temporal discretization for $\Delta t_{\text {aggr }}^{*} \leq 10^{-2}$ and it is at the same levels of Fig. 7a. Therefore, for a particle depletion level of 80\%, an $0-2 \%$ concentration error is due to the MC methodology. As opposed to $n_{\text {int }}$, a coarse temporal discretization (i.e. the equivalent of $\Delta t_{\text {aggr }}^{*} \approx 10^{-1}$ ) has a pronounced effect on the evolution of the particle concentration. The aggregation rate is overestimated leading to an underestimation of the particle concentration $N_{c}$. Arguably, a more conservative limit for the time-step than Eq. (22) should be used when the kernels are also a function of the particle 

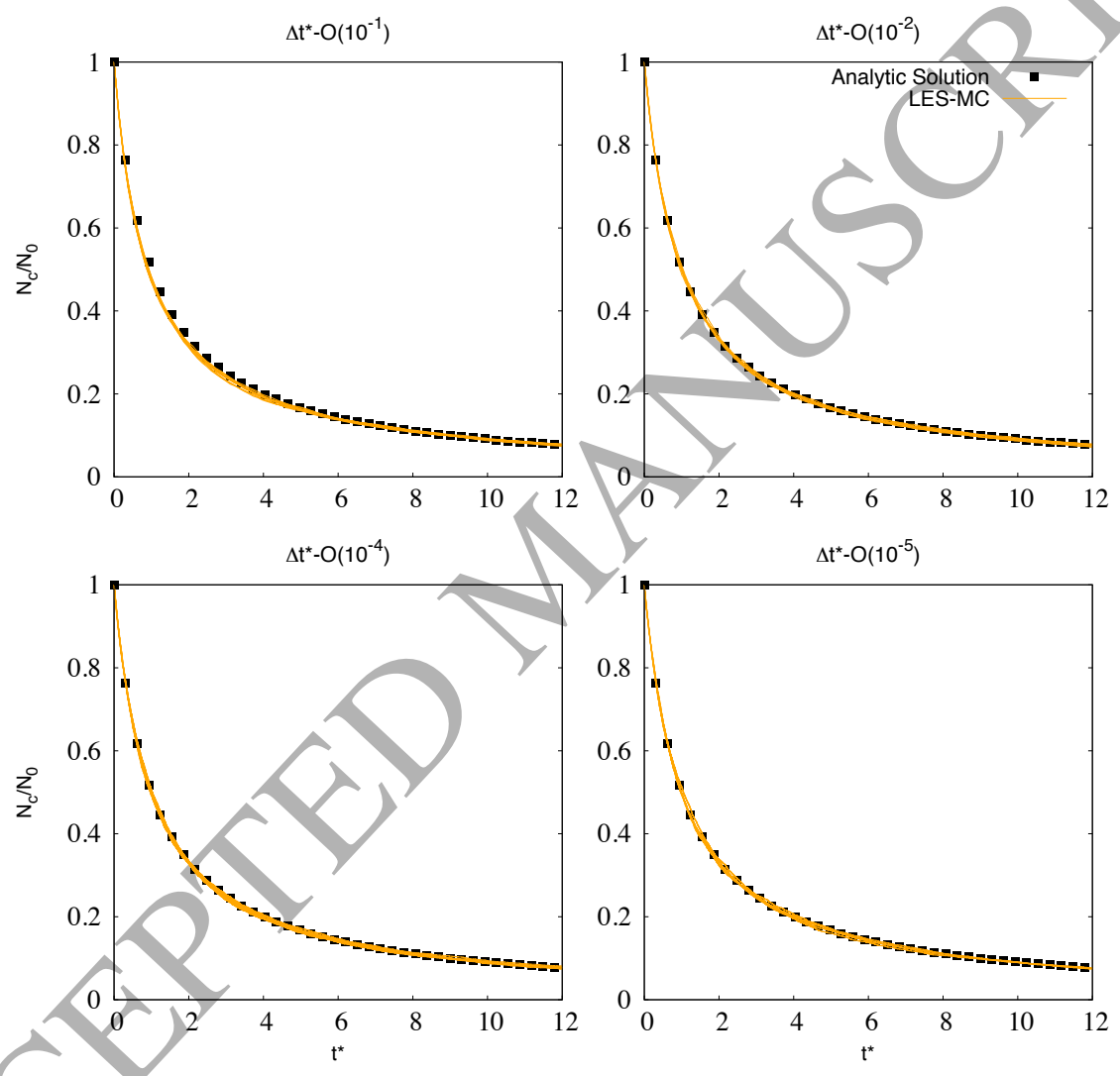

Figure 8: Total particle concentration versus dimensionless time for the same initial particle concentration using $\Delta t_{\text {aggr }}^{*}=10^{-1}, 10^{-2}, 10^{-4}$, and $10^{-5}$ and with $n_{0}^{*}=1.0 \%$. 


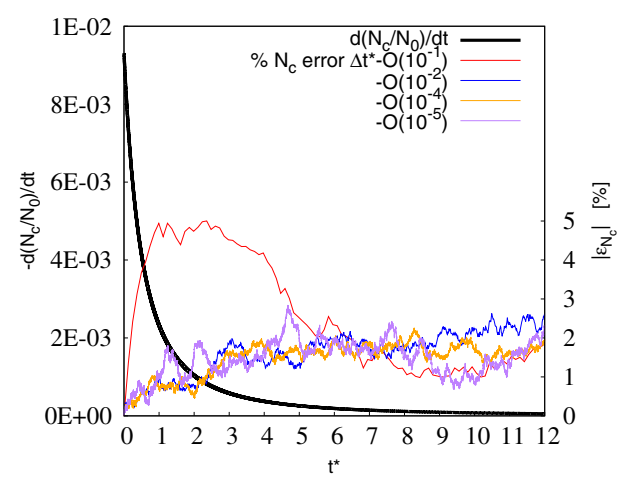

(a) Rate of particle depletion (left) Concentration error (right).

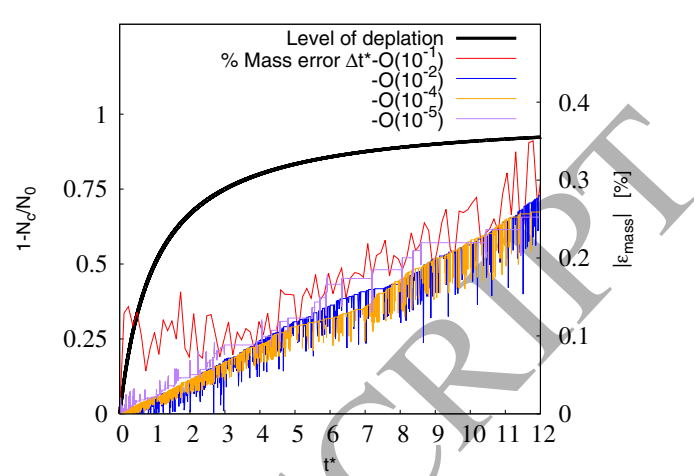

(b) Level of particle depletion (left) Mass error (right).

Figure 9: Error levels for different temporal discretization.

volume.

Figure 9b shows the error in total particle mass for different aggregation time-steps. Qualitatively the error shows a similar monotonic increase as see in Fig. 7b. Nevertheless, mass errors are at negligible levels for both temporal and parcelinternal particle number discretization.

\subsection{Evolution of the Particle Size Distribution}

The model has exhibited good agreement with mean population values, with only a weak dependency on the choice of the initial $n_{\text {int }}$ and $\Delta t_{\text {aggr }}^{*}$. However, it is important to investigate the effect of the aforementioned parameters with respect to the evolution of a full PSD. To this end, analytic solutions for the temporal evolution of the PSD of an aggregating population of particles are used. An initial PSD is assumed:

$$
\left.\mathcal{N}(v)\right|_{t=0}=\frac{N_{0}}{v_{0}} \exp \left(-v / v_{0}\right)
$$


where $v_{0}$ is the initial count mean particle volume.

To enable a comparison between different $n_{\text {int }}$, the initial particle population is constant and equal to $N_{0} V_{\text {cell }}=1000$. For every $n_{\text {int }}$-case investigated, the simulation is repeated 450 times until the total number of initialised real particles is equal to $4.5 \cdot 10^{5}$. Note that the number of total parcels/samples initialised decreases with $n_{\text {int }}$, i.e. a total of $0.018,0.18,1.8$ and $4.5 \cdot 10^{5}$ parcels are initialised for the $n_{0}^{*}=25 \%, 2.5 \%, 0.25 \%$ and $n_{\text {int }}=1$ cases, respectively. The resulting PSDs from a given case are constructed from the cumulative number of parcels present at the prescribed time-instances after every simulation.

This cumulative sampling can be considered representative of the PSD of a total $s_{n}$ cells used to discretize a domain with a given initial particle concentration $N_{0}$ and particle size distribution $\mathcal{N}(v, 0)$.

To quantify the error, the cumulative Sauter Mean Diameter (SMD) is calculated for every simulation as:

$$
d_{32}=\frac{\sum_{k=1}^{\infty} \mathcal{N}\left(v_{k}\right) v_{k}}{\sum_{k=1}^{\infty} \mathcal{N}\left(v_{k}\right) v_{k}^{2 / 3}}
$$

and it represents a higher-order moment ratio with the dimensions of particle diameter.

\subsubsection{Constant kernel $\beta_{i j}=\beta_{c}$}

Scott (1968) derived analytically the solution for Equation (23), where the PSD as a function of time for a constant aggregation kernel $\beta_{c}$ is given by:

$$
\mathcal{N}(v, t)=\frac{4 N_{0}}{v_{0}\left(\beta_{c} N_{0} t+2\right)^{2}} \exp \left[\frac{-2 v}{v_{0}\left(\beta_{c} N_{0} t+2\right)}\right]
$$


For simplicity the initial concentration $N_{0}$, particle mean volume $v_{0}$, and aggregation kernel $\beta_{c}$ are chosen to be 1 .

Following the work of Rigopoulos and Jones (2003), the PSD is evolved in time for 10 and 20 seconds $\left(t^{*}=5,10\right)$. These residence times correspond to depletion levels of $82 \%$ and $90 \%$. The time discretization is fixed to $\Delta t_{\text {aggr }}^{*}=$ $10^{-4}$ and the initial parcel concentration $n_{\text {int }}$ is varied.

Figure 10 presents the PSD at $t=0,10$ and $20 \mathrm{~s}$ for four choices of initial parcel internal population, namely: $n_{0}^{*}=25 \%, 2.5 \%, 0.25 \%$ and $n_{\text {int }}=1$. From an order of magnitude perspective the model performs well independently of the choice of $n_{\text {int }}$. For $n_{0}^{*} \leq 0.25 \%$ the PSD agrees very well with the analytic equivalent at the times considered. For larger internal parcel populations $\left(n_{0}^{*}=2.5 \%\right.$ and $\left.25 \%\right)$ there is a bias towards larger sizes, and a non-physical peak is found at $v \approx 10$.

The source of this discrepancy depends on the intrinsic error in the solution procedure, but also on the error in the initial PSD discretization. As noted, this error relates to the total number of samples (i.e. parcels) used to discretize the size distribution of the initialised real particle population. In the case of Fig. 10, where each parcel represents $25 \%$ of the concentration $N_{0}$, the 'linear' sets of points at $t=0$ represent exactly the aforementioned error. Every yolume in the range of $v / v_{0}=6 \rightarrow 10$ is, for example, represented by a single parcel. If the initial PSD is not captured accurately, the errors propagate to the subsequent distributions as the aggregation process progress.

Figure 11 summarises the percentage absolute error in the simulated SMD for the various initial $n_{i n t}$ and aggregation residence times. In all of the 

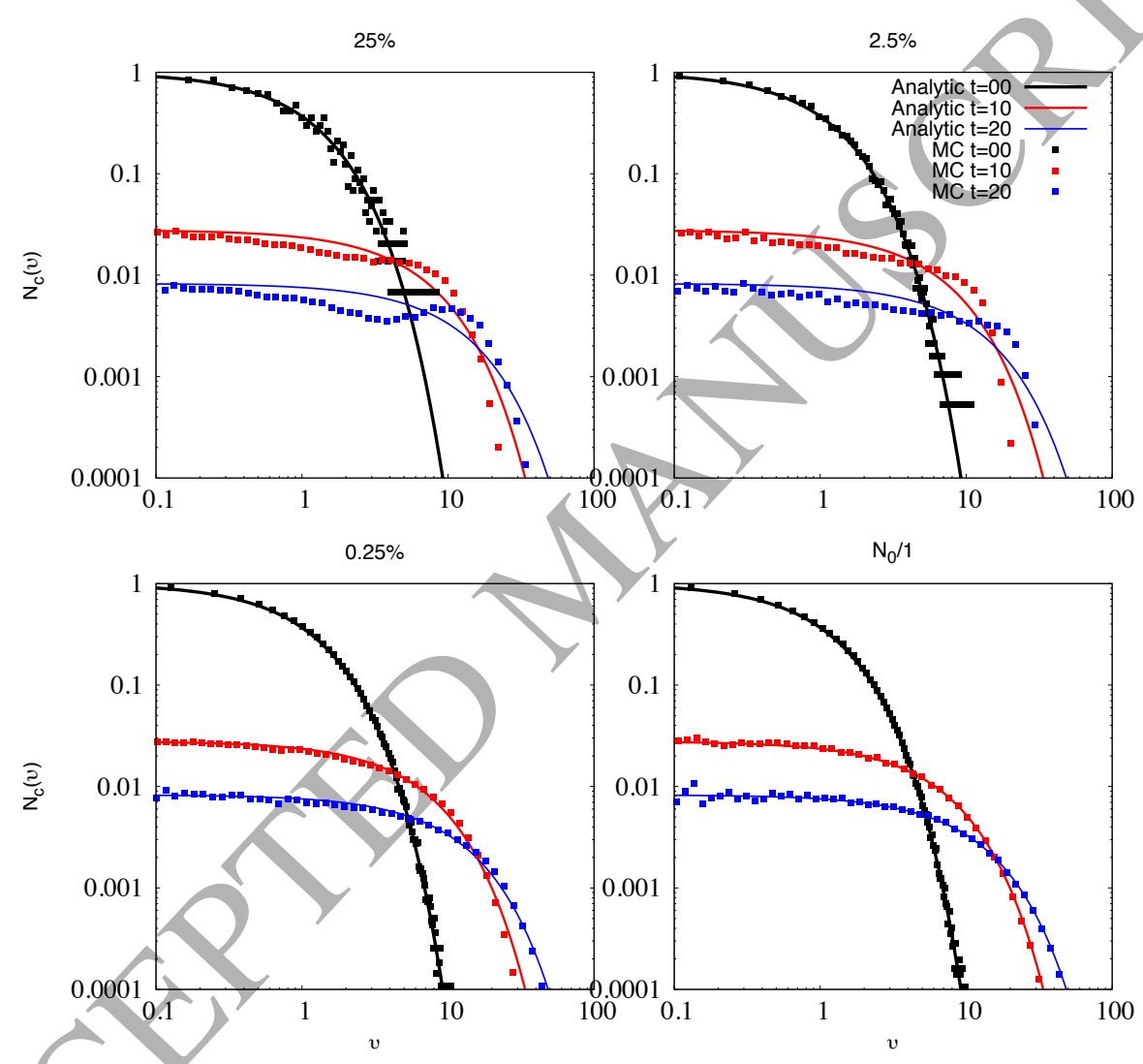

Figure 10: Evolution of the PSD for an initial exponential distribution for $n_{0}^{*}=25 \%$, $2.5 \%, 0.25 \%$ and $n_{\text {int }}=1$. 


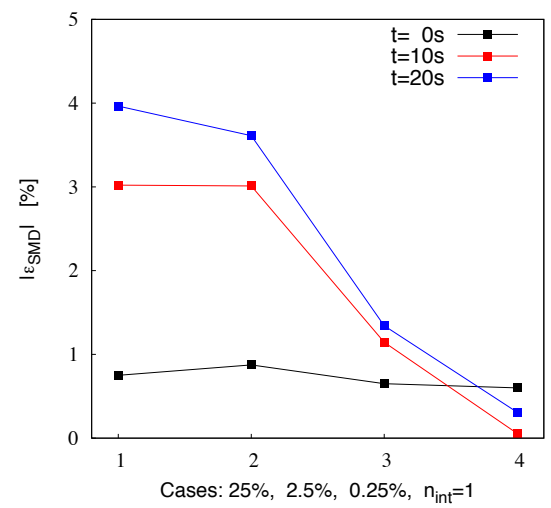

Figure 11: Relative error in the SMD for three residence times $t=0,10$, and 20 s for different cases of initial parcel internal population.

cases the SMD of the initial PSD converges to less than $1 \%$ of the analytic equivalent. At aggregation residence times of $t=10$ and $20 \mathrm{~s}$, the coarsest cases where $n_{0}^{*} \geq 2.5 \%$ exhibit an error which does not exceed $\left|\epsilon_{S M D}\right| \approx 4 \%$. In the finer cases $\left(n_{0}^{*}=0.25 \%\right.$ and $\left.n_{i n t}=1\right)$ the errors are $\left|\epsilon_{S M D}\right| \leq 1.3 \%$.

Finally, from Figs 10 and 11 , a choice of $n_{0}^{*} \sim \mathcal{O}\left(10^{-1 \%}\right)$ reproduces accurately the analytic solution for the PSD evolving with a constant kernel.

For $n_{0}^{*}=25 \%$ the eyolution of the mean particle concentration and SMD are captured with an error of less than $2.5 \%$ and $4.5 \%$, respectively.

\subsubsection{Golovin kernel $\beta_{i j}=\beta_{c}\left(v_{i}+v_{j}\right)$.}

The Golovin kernel $\beta_{i j}=\beta_{c}\left(v_{i}+v_{j}\right)$ is proportional to the interacting particle volumes. Golovin (1963) derived an analytic solution for an initial particle population with exponentially-distributed volumes; see Eq. (23). The temporal evolution of the PSD with the aforementioned initial condition 
reads:

$$
\mathcal{N}(v, t)=\frac{(1-\theta) \exp \left[-\frac{v}{v_{0}}(\theta+1)\right]}{\frac{v}{v_{0}} \theta^{1 / 2}} \mathrm{I}_{1}\left(2 \frac{v}{v_{0}} \theta^{1 / 2}\right),
$$

where $\theta=1-\exp \left(-\beta_{c} N_{0} v_{0} t\right)$ and $\mathrm{I}_{1}$ is the first order Bessel-I function. Same parameters as the constant kernel case have been chosen. The PSD is evolved in time for 0.5 and $2.0 \mathrm{~s}$ (as in the work of Rigopoulos and Jones (2003)), equivalent to a $40 \%$ and an $86 \%$ particle depletion. The temporal discretization is fixed to a normalized time-step of $\Delta t_{\text {aggr }}^{*}=10^{-6}$ (based on $v_{i}+v_{j}=1$ ), which was found sufficient to uncouple the aggregation process. As opposed to the constant kernel, a large number of simulations were performed such that the initial PSD is accurately captured, within $\left|\epsilon_{S M D}\right| \leq 0.5 \%$, in order to isolate the aggregation model performance.

Figure 12 shows the PSD for three residence times and four cases of initial parcel internal population, the results agree very well with the analytic solution. The $n_{0}^{*}=25 \%$ case reproduces accurately the initial distribution and the evolved PSD at $t=0.5 \mathrm{~s}$. However, at $t=2 \mathrm{~s}$ the number of smaller particles are slightly over-estimated. For the smaller $n_{0}^{*}$ cases the model captures the analytic PSD at all times.

When compared to the results from the constant kernel (Fig. 10), the resolution of the initial particle distribution is very important as it sets the boundary gondition for the evolution of the PSD. In addition, in the case of the constant kernel, small particles aggregate with the same kernel-value as larger particles, and due to their higher concentration smaller particles aggregate with a higher probability than larger ones. This leads to a more uniform distribution (depletion) of sizes in the range of $v / v_{0}<10$. On the other hand, the Golovin kernel has a non-linear effect on the PSD, as 

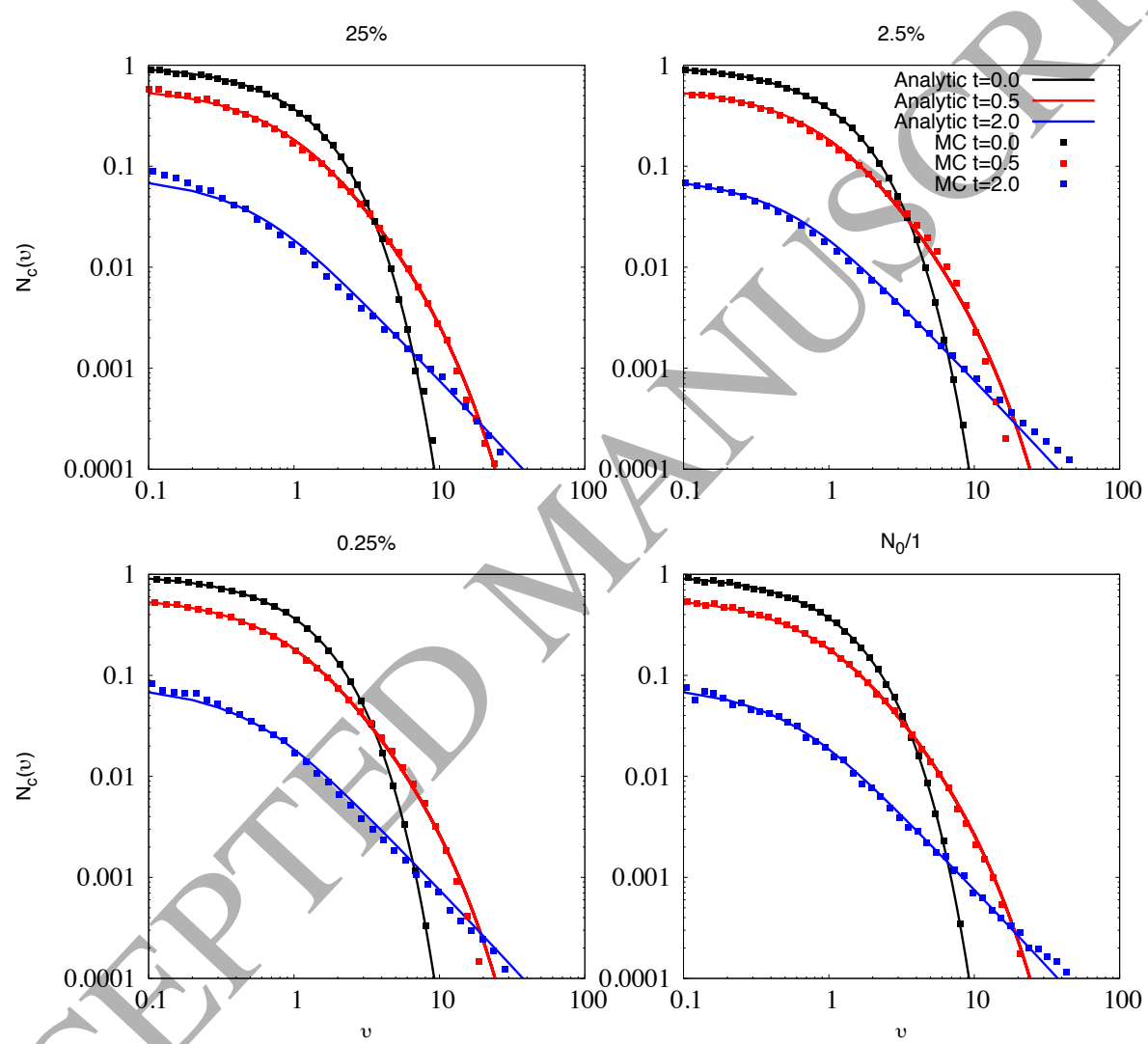

Figure 12: Evolution of the PSD with the Golovin kernel for an initial exponential distribution and $n_{0}^{*} V_{\text {cell }}=25 \%, 2.5 \%, 0.25 \%$ and $n_{\text {int }}=1$. 


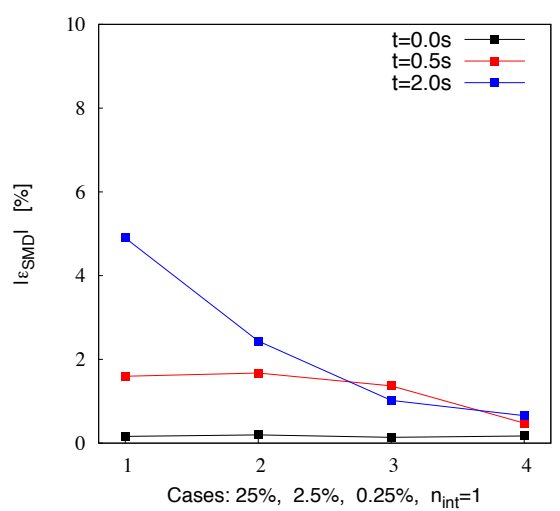

Figure 13: Relative error in the SMD for three residence times $t=0,0.5,2 \mathrm{~s}$ for different cases of initial parcel internal population $\left(n_{0}^{*}=25 \%, 2.5 \%, 0.25 \%\right.$ and $n_{\text {int }}=1$, numbered one to four, respectively)

larger particles have higher kernel values. The combined effect leads to a less uniform decay of populations. In fact the smallest class, where $v=0.1$, has a density of 0.07 after an 86\% particle depletion, whereas the density from the constant kernel after an $82 \%$ depletion level is 0.03 . In the Golovin kernel there is an intrinsic directionality in the aggregation process, where smaller particles tend to aggregate more with larger particles rather than with particles of similar volumes. This process is inherently captured in the present aggregation model, where small particles aggregate with large particles and so forth.

Figure 13 shows the resulting errors in the SMD calculated using Eq. (24). For a residence time of $t=0.5 \mathrm{~s}$ the SMD is captured within an error of $\left|\epsilon_{S M D}\right|<2 \%$ and for $t=2.0 \mathrm{~s}$ the SMD is predicted within $\left|\epsilon_{S M D}\right|<1 \%$ for the two fine cases. The coarser cases have approximately the same level of error after $t=0.5 \mathrm{~s}$ as only a small amount of the initial particle population 
has been depleted. For a longer residence time $(t=2.0 \mathrm{~s})$ the error in the SMD increases for the coarsest cases. Nevertheless, even for the coarsest case where $n_{0}^{*}=25 \%$, the model predicts the shape of the PSD as seen in Fig. 12 with a relatively small error.

\section{Conclusions}

In the present work, a new stochastic model has been presented to simulate the aggregation process. The proposed model allows for a simple multicollision implementation, where each particle of a numerical parcel can aggregate with any other parcel of a 'computational cell. The model can be directly coupled to an unsteady CFD solution.

In a CFD-coupled simulation, the aggregation event is modelled for every time-step and computational cell. The concentration and properties of a parcel are updated from the previous time step with the corresponding spatial dispersion.

The model was validated against analytic solutions of different aggregation kernels, and it was shown that the inclusion of internal collisions for every parcel is required to accurately represent the particle depletion process. The mass errors are at negligible levels for both temporal and parcel internal-particle-number discretization. In addition, a heuristic stability condition was suggested in Eq. (22) to de-couple the aggregation process from the temporal discretization.

The model reproduces the analytic PSD evolution using both the constant and the Golovin aggregation kernels, with SMD errors below $5 \%$ in the worse scenarios, even if the PSD extends four orders of magnitude in both class and 
particle density. The results suggested that an initial particle population of $n_{\text {int }} \approx 0.25 N_{0} V_{\text {cell }} \%$ is necessary to resolve the evolution of the PSD with a constant kernel. Higher resolution could be obtained by appropriately discretizing the PSD regions with lower densities.

\section{References}

Adam, J., Lindblad, N., 1968. The collision, coalescence, and disruption of water droplets. J. Appl. Phys. 39, 173.

Ashgriz, N., Poo, J., 1990. Coalescence and separation in binary collision of liquid drops. J. Fluid Mech. 221, 183.

Azizi, F., Taweel, A. A., 2010. Algorithm for the accurate numerical solution of PBE for drop breakup and coalescence under high shear rates. Chem. Eng. Sci. 65, 6112-6127.

Bini, M., 2006. Large Eddy Simulation of particle and droplet laden flows with stochastic modelling of subfilter scales. Ph.D. thesis, Imperial College London.

Brauer, M., Hoek, G., Vliet, P. V., Meliefste, K., Fischer, P., Wijga, A., Koøpman, L., Neijens, H., Gerritsen, J., Kerkhof, M., Heinrich, J., Bellander, T., Brunekreef, B., 2002. Air pollution from traffic and the development of respiratory infections and asthmatic and allergic symptoms in children. A. J. Respiratory and Critical Care Medicing 8, 1092-1098.

Eibeck, A., Wagner, W., 2003. Stochastic interacting particle systems and nonlinear kinetic equations. Ann. Appl. Probab. 13, 845-889. 
Finlayson-Pitts, B., Pitts, J., 1997. Tropospheric air pollution: ozone, airborne toxics, polycyclic aromatic hydrocarbons, and particles. Science 276, $1045-1052$.

Friedlander, S., 2000. Smoke, Dust, and Haze: Fundamentals of Aerosol Dynamics. Oxford University Press.

Gavaises, M., Theodorakakos, A., Bergeles, G., Brenn, G., 1996. Evaluation of the effect of droplet collisions on spray mixing. Proe. Instn Mech. Engrs $210,465-475$.

Golovin, A., 1963. The solution of the coagulation equation for cloud droplets in a rising air current. Bull. Acad. Sci. SSSR Geophys. Ser. (English Transl.), 482-487.

Guo, B., Fletcher, D., Langrish, T., 2003. Simulation of the agglomeration in a spray using lagrangian particle tracking. Applied Mathematical Modelling 28, 273-290.

Ho, C., Sommerfeld, M., 2002. Modelling of micro-particle agglomeration in turbulent fows. Chem. Eng. Sci. 57, 3073-3084.

Johannessen, T., Pratsinis, S., Livbjerg, H., 2001. Computational analysis of coagulation and coalescence in the flame synthesis of titania particles. Powder Tech. 118, 242-250.

Klimontovich, I., 1969. The Statistical Theory of Non-equilibrium Processes in a Plasma. Pergamon. 
Koch, D., Pope, S., 2002. Coagulation-induced particle-concentration fluctuations in homogeneous, isotropic turbulence. Phys. Fluids 14(7), 24472455 .

Li, Z., Wang, H., 2003. Drag force, diffusion coefficient, and electric mobility of small particles. i. theory applicable to the free-molecule regime. Phys. Rev. E, 061206.

Liao, Y., Lucas, D., 2010. A literature review on mechanisms and models for the coalescence process of fluid particles. Chem. Eng. Sci 65, 2851-2864.

Liffman, K., 1992. A direct simulation Monte-Carlo method for cluster coagulation. J. Comput. Phys. 100, 116-127.

Lin, Y., Lee, K., Matsoukas, T., 2002. Solution of the population balance equation using constant-number Monte Carlo. Chem. Eng. Sci. 57(12), $2241-2252$.

Litchford, R., Jeng, S., 1991. Efficient statistical transport model for turbulent particle dispersion in sprays. AIAA Journal 29, 1443.

Matveev, S. A., Smirnov, A., Tyrtyshnikov, E., 2015. A fast numerical method for the Cauchy problem for the Smoluchowski equation. Journal of Computational Physics 282, 23-32.

Maximova, N., Dahl, O., 2006. Environmental implications of aggregation phenomena: Current understanding. Current Opinion in Colloid \& Interface Science 11, 246-266. 
Miller, S., Garrick, S., 2004. Nanoparticle coagulation in a planar jet. Aerosol Sci. Tech. 38, 79-89.

Montes, I., Rego, G., Camblor, C., Quero, A., Gonzalez, A., Rodriguez, C., 2004. Respiratory disease in aggregate quarry workers related to risk factors and Pi phenotype. J. Occup. Environ. Med. 46(11), 1150-1157.

Nijdam, J., Guo, B., Fletcher, D., Langrish, T., 2004. Challenges of simulating droplet coalescence within a spray. Drying Tech. 22(6), 1463-1488.

Oberdorster, G., 2001. Pulmonary effects of inhaled ultrafine particles. Int. Arch. Occup. Environ. Health 74, 1-8.

Patterson, R., Wagner, W., Kraft, M., 2011. Stochastic weighted particle methods for population balance equations. J. Comput. Phys. 230(19), $7456-7472$.

Pesmazoglou, I., Kempf, A., Navarro-Martinez, S., 2013. A dynamic model for the lagrangian stochastic dispersion coefficient. Phys. Fluids 25, 125108.

Pesmazoglou, I. Kempf, A., Navarro-Martinez, S., 2014. Aerosol nucleation in a turbulent jet using large eddy simulations. Chem. Eng. Sci. 116, 383397.

Rigopoulos, S., 2010. Population balance modelling of polydispersed particles in réactive flows. Prog. Energy Combust. Sci. 36, 412-443.

Rigopoulos, S., Jones, A., 2003. Finite-element scheme for solution of the dynamic population balance equation. AICHE J 49, 1127-1139. 
Rosner, D., McGraw, R., Tandon, P., 2003. Multi-variate population balances via moment and Monte Carlo simulation methods. Ind. Eng. Chem. Res. 42, 2699-2711.

Ruger, M., Hohmann, S., Sommerfeld, M., Kohnen, G., 2000. Euler/Lagrange calculations of turbulent sprays: the effect of droplet collisions and coalescence. Atomization and Sprays 10(1), 47-81.

Scott, W., 1968. Analytic studies of cloud droplet coalescence. J. of Atmospheric Sciences 25, 54-65.

Smith, M., Matsoukas, T., 1998. Constant-number Monte Carlo simulation of population balances. Chem. Eng. Sci. 53(9), 1777-1786.

Smoluchowski, M., 1917. Versuch einer mathematischen theorie der koagulationskinetik kolloider lsungen. Z. Phys. Chem. 92, 129168.

Sommerfeld, M., 2001. Validation of a stochastic lagrangian modelling approach for inter-particle collisions in homogeneous isotropic turbulence. Int. J. Multiphase Flow 27, 1829-1858.

Sundaram, S., Collins, L., 1997. Collision statistics in an isotropic particleladen turbulent suspesion. Part 1. Direct numerical simulations. J. Fluid Mech. 335, 75-109.

Villermaux, J., Devillon, J., 1972. Representation de la coalescence et de la re-dispersion des domaines de segregation dans un modele d'interaction phenomenologique. In: Proceedings of the Second International Symposium on Chemical Reaction Engineering, pages 1-13, Elsevier, New York. 
Wagner, W., 2003. Stochastic, analytic and numerical aspects of coagulation processes. Mathematics and Computers in Simulation 62, 265-275.

Williams, F., 1958. Spray combustion and atomisation. Phys. Fluids 1 n.6, $541-545$.

Yamamoto, Y., Potthoff, M., Tanaka, T., Kajishima, T., Tsuji, Y., 2001. Large-eddy simulation of turbulent gas-particle flow in a vertical channel: effect of considering inter-particle collisions. J. Fluid Mech. 442, 303-334.

Yan, J., Luo, K., Fan, J., Tsuji, Y., Cen, K., 2008. Direct numerical simulation of particle dispersion in a turbulent jet considering inter-particle collisions. Int. J. Multiphase Flow 34, 723-733.

Yeoha, G., Tub, J., 2006. Two-fluid and population balance models for subcooled boiling flow. Appllied Mathematical Modelling 30(11), 1370-1391.

Yu, M., Lin, J., 2010. Nanoparticle-laden flows via moment method: A review. Int. J. Multiphase Flow 36, 144-151.

Zhao, H., Kruis, F., Zheng, C., 2009. Reducing statistical noise and extending the size spectrum by applying weighted simulation particles in Monte Carlo simulations of coagulation. Aerosol Sci. Tech. 43, 781-793.

Zhao, H. Zheng, C., 2013. A population balance-Monte Carlo method for partícle coagulation in spatially inhomogeneous systems. Computers \& Fluids 71, 196-207.

Zheng, H. Z. C., 2011. Two-component brownian coagulation: Monte Carlo simulation and process characterization. Particuology 9, 414-423. 
Zou, Y., Kavousanakis, M., Kevrekidis, I., Fox, R., 2010. Coarse-grained computation for particle coagulation and sintering processes by linking quadrature method of moments with monte-carlo. J. Comp. Phys. 229, $5299-5314$.

Zucca, A., Marchisio, D., Baressi, A., Fox, R., 2006. Implementation of the population balance equation in cfd codes for modelling soot formation in turbulent flames. Chem. Eng. Sci. 61, 87-95. 